\title{
BIENESTAR EN VACAS EN EL PREPARTO DURANTE EL VERANO 2014 EN EL DEPARTAMENTO CASTELLANOS. ESTUDIO DE CASO
}

\author{
Leva, P. E. ${ }^{1} ;$ Toneatti, I. E. ${ }^{1} ;$ Stegmayer, M. I. ${ }^{1}$ Ghiano, J. ${ }^{2}$; \\ Toffoli, G. D. ${ }^{1}$; Fernandez, G. ${ }^{1,3}$; Garcia, M. S. ${ }^{1} \&$ Sosa, J. L. ${ }^{3}$
}

\begin{abstract}
RESUMEN
Durante el verano del 2014 se llevó a cabo un estudio de caso con el objetivo de evaluar algunas respuestas fisiológicas y conductuales preparto y productivas postparto, en vacas lecheras de alto merito genético con acceso a sombras artificiales. Se utilizaron 24 vacas en preparto que se alojaron en un corral seco. Las sombras estaban ubicadas en el área de descanso y en el patio de comida. El peso corporal y la condición corporal fueron evaluadas al inicio y a los 30 días de comenzado el ensayo. La frecuencia respiratoria se midió dos veces por semana en tres momentos del día. El registro de comportamiento una vez a la semana. El peso corporal y condición corporal aumentaron durante este periodo indicando que la combinacion de dieta adecuada con la provisión de sombra fue favorable. La frecuencia respiratoria en promedio fue de $60 \mathrm{rpm}$, indicando un estrés medio alto. La conducta de parado a la sombra fue la de mayor prevalencia. La conducta de comer se mantuvo dentro de los valores considerados normales: 92 minutos. La producción lechera fue de entre 30 y 35 1/d, normal para el establecimiento. El índice de temperatura y humedad se mantuvo por encima de 72. Además, durante febrero las lluvias fueron superiores a las normales de la zona, ocasionado problemas de piso en los corrales.
\end{abstract}

Palabras claves: Respuestas fisiologicas, produccion lechera, comportamiento, indice de temperatura y humedad.

\begin{abstract}
Characterization of Farmers Markets in the Province of Córdoba (Argentina).

During the summer of 2014 it was carried out a case study in order to evaluate some physiological and behavioral prepartum and postpartum production in dairy cows of high genetic merit responses with access to artificial shades were used. 24 cows in prepartum they stayed in a dry pen. The shadows were located in the rest area and food court. Body weight and body condition were evaluated at start and 30 days into the trial. The respiratory rate was held twice a week in
\end{abstract}

1.- Facultad de Ciencias Agrarias (UNL). Kreder 2805. (3080) Esperanza, provincia de Santa Fe. Email: pleva@fca.unl.edu.ar

2.- EEA Inta Rafaela. Ruta 34 - Km 227. (2300) Rafaela, provincia de Santa Fe.

3.- Facultad de Ciencias Veterinarias (UNL). Kreder 2805. (3080) Esperanza, provincia de Santa Fe.

Manuscrito recibido el 28 de abril de 2015 y aceptado para su publicación el 4 de diciembre de 2015. 


\section{P. E. Leva et al.}

three times a day. The behavior log once a week. Body weight and body condition gain during this period indicating adequate diet was combined with the provision of shade. The average respiratory rate was $60 \mathrm{rpm}$, indicating a high middle stress. The conduct of standing in the shadow was the most prevalent. Eating behavior are kept within normal values: 92 minutes. Milk production was between 30 and $351 / \mathrm{d}$, normal setting. The temperature and humidity index remained above 72 . In addition, during february the rains were above normal in the area, caused problems in floor pens. Key words: Physiological responses, milk production, behavior, temperature and humidity index.

\section{INTRODUCCIÓN}

La producción animal en general debe considerar el bienestar como uno de los aspectos importantes. El concepto de bienestar animal (BA) es complejo. No existe una definición universal, sino varias desde distintas aproximaciones. Una de las más aceptadas es la de Broom (1996), que define al BA como el estado en el cual el animal es capaz de enfrentar y adaptarse al ambiente y a los cambios que en él se producen. De la definición anterior, se deduce que el BA es un estado propio del animal y puede ser estimado por la medición de los esfuerzos que realiza el animal para alcanzar el estado ideal. Si un animal no puede satisfacer una necesidad determinada tendrá poco bienestar.

Dentro del sistema productivo existe un período del ciclo evolutivo de las vacas lecheras durante el cual es poca la importancia que se le da al bienestar animal: la etapa de vaca en preparto. Esta categoría es bastante relegada en relación con el manejo ambiental durante la época estival por considerarse una categoría improductiva. Sin embargo, este período de transición a la lactancia (pre y pos-parto inmediatos) es crítico para la salud del ganado lechero (12). El estrés calórico puede reducir los niveles de hormonas tiroideas y estrógenos placentales, mientras que incrementa los niveles en sangre de ácidos grasos no esterificados (NEFA) lo cual puede alterar el desarrollo de la ubre y la placenta y la producción de leche entregada al ternero (6). Considerando que el período seco es la etapa de preparación de la vaca para su próxima lactancia y que el pico de producción se alcanza a las 8 semanas postparto, la etapa previa al parto toma gran importancia.

Además, es bien sabido que durante la época estival hay una disminución del consumo voluntario $(45,46,47)$. Pero por otro lado, aumentan los requerimientos nutricionales de mantenimiento como mecanismo para mantener el balance térmico y la homeostasis. Fox et al. (1988) indicaron que los requerimientos de mantención del ganado fluctúan entre los valores recomendados por el NRC (1981) sin estrés y cuatro veces dicho valor bajo condiciones ambientales extremas. Así, dentro de la zona de termoneutralidad, la energía de la dieta es utilizada para mantención, crecimiento, producción de leche y actividad física; mientras que bajo o sobre la zona de termoneutralidad la energía es reorientada a funciones tendientes a mantener la condición homeotérmica y en algunos casos puede existir un aumento en la demanda de energía para estos procesos (11).

Por lo tanto, la introducción de manejos estratégicos que permitan mejorar las condiciones ambientales podrían disminuir el 